\title{
Short-term Comparison of Supervised Rehabilitation and Home- based Rehabilitation for Earlier Recovery of Shoulder Motion, Pain, and Function after Rotator Cuff Repair
}

\author{
Si-Jung Song*, Tae-Ho Jeong*, Jung-Wha Moon, Han-Vit Park, Si Yung Lee, Kyoung-Hwan Koh \\ Department of Orthopaedic Surgery, Inje University Ilsan Paik Hospital, Goyang, Korea
}

Background: This study was undertaken to compare the outcome of supervised and home exercises with respect to range of motion (ROM), pain, and Single Assessment Numeric Evaluation (SANE). We further correlated the ROM recovery and pain reduction as well. Methods: The study included 49 patients who underwent arthroscopic rotator cuff repair. Rehabilitation was initiated after 4 weeks of immobilization. A total of 29 patients performed supervised exercise 3 times a week. Standardized education and brochures for review were provided to the remaining 20 patients who insisted on home rehabilitation. Statistical analysis was performed for comparing pain Numerical Rating Scale (NRS), SANE, and ROM. In addition, we also evaluated the correlation between pain and ROM.

Results: Comparison of the two groups revealed no significant differences in forward flexion, internal rotation, abduction, and pain NRS. However, SANE at the 9 th week (63.8 vs. 55.0, $p=0.038$ ) and improvement of external rotation from the 5th to the 9th week (17.6 vs. 9.3, $p=0.018$ ) were significantly higher in the supervised exercise group as compared to the home exercise group. Correlation of pain NRS with forward flexion, external rotation, internal rotation and abduction were statistically not significant (correlation coefficient $=0.032$ [ $p=0.828],-0.255$ [ $p=0.077], 0.068$ [ $p=0.642]$, and -0.188 [ $p=0.196]$, respectively).

Conclusions: The supervised rehabilitation after arthroscopic rotator cuff repair showed better improvement in external rotation and higher SANE score after 4 weeks of rehabilitation exercise. However, no statistically significant correlation was observed between the recovery of ROM and short-term pain relief.

(Clin Shoulder Elbow 2018;21(1):15-21)

Key Words: Rotator cuff; Rehabilitation; Range of motion

\section{Introduction}

The clinical outcomes of an arthroscopic rotator cuff repair differ depending on the rehabilitation exercise after surgery, in addition to age, the size of rupture, and the proper suture method. ${ }^{1,2)}$ Without appropriate rehabilitation exercise, successful repairs could also result in unsatisfactory outcomes, such as limitation in range of motion (ROM), persistent pain, and occasional retear. Since rehabilitation exercises usually begin after a certain period of immobilization post repair, proper rehabilita- tion is essential for the early recovery of ROM, pain control, and healing of the repaired tendon. ${ }^{3)}$ Inappropriate pain control and persistent limitations in ROM after the surgery could delay an early return to the daily activities, thereby decreasing the quality of life, at least in the short-term. In addition, uncontrolled shoulder motion exercise might lead to a retear of the tendon. ${ }^{4}$

Therefore, post repair rehabilitation and a certain period of immobilization is emphasized for patients. Rehabilitation can be performed as a home-based exercise program or a supervised exercise program in a hospital. Since supervised rehabilitation

Received September 15, 2017. Revised November 8, 2017. Accepted January 25, 2018.

*These authors contributed equally to this article as co-first authors.

Correspondence to: Kyoung-Hwan Koh

Department of Orthopaedic Surgery, Inje Universtiy Ilsan Paik Hospital, 170 Juhwa-ro, Ilsanseo-gu, Goyang 10380, Korea

Tel: +82-31-910-7995, Fax: +82-31-910-7967, E-mail: osdoc.koh@gmail.com

IRB approval: Inje University Ilsan Paik Hospital (No. ISPAIK 2016-12-033-001).

Financial support: None. Conflict of interests: None. 
exercises may especially be unavailable due to temporal or financial causes of the patient, home-based rehabilitation exercise may be conducted after imparting appropriate education. Lisinski et al. ${ }^{5)}$ reported better pain management and ROM after arthroscopic rotator cuff repair in an exercise group supervised by experts. Other studies also reported that in conservative treatment of rotator cuff lesions, the supervised exercise group showed better results ${ }^{6-8)}$ as well as lower retear rates. ${ }^{9)}$ On the other hand, Büker et al. ${ }^{10)}$ reported no difference in the clinical outcomes between supervised rehabilitation and home exercise groups after an arthroscopic rotator cuff repair, which was corroborated by another study with similar results. ${ }^{11)}$ Hence, there are diverse results in literature when comparing the mode of rehabilitation exercises after arthroscopic rotator cuff repair.

Since all prior reports consider the outcomes after a relatively long-term follow-up, it is hard to find studies reporting the shortterm effects of the rehabilitation protocols, especially the early recovery of motion arc and pain. Pain relief and recovery of motion arc in the short-term could be important factors related to patient satisfaction and their quality of life, although the longterm prognosis is of utmost importance.

This study was therefore undertaken to compare the shortterm effects of supervised rehabilitation and home exercise. Our null hypothesis suggests that the two rehabilitation programs are not different in terms of ROM gain, pain relief, and functional recovery. In addition, we further investigated the correlation between ROM gain and pain relief.

\section{Methods}

This study was approved by the Institutional Review Board of Inje University Ilsan Paik Hospital (IRB No. ISPAIK 2016-12-033001).

\section{Patients}

This study was conducted by recruiting patients who agreed to participate in the rehabilitation exercises following arthroscopic rotator cuff repair. All repairs were performed by a single shoulder specialist, from June 2015 through February 2017. Revision surgery, combined adhesive capsulitis before surgery, history of glenohumeral dislocation or fracture around the shoulder joint, accompanying inflammatory arthritis and calcific tendinitis, Lafosse classification ${ }^{12)}$ type II, or higher subscapularis tear cases were excluded from the subjects. A supervised rehabilitation exercise program was advised for all patients. Patients who wanted home rehabilitation exercise despite the prescription of
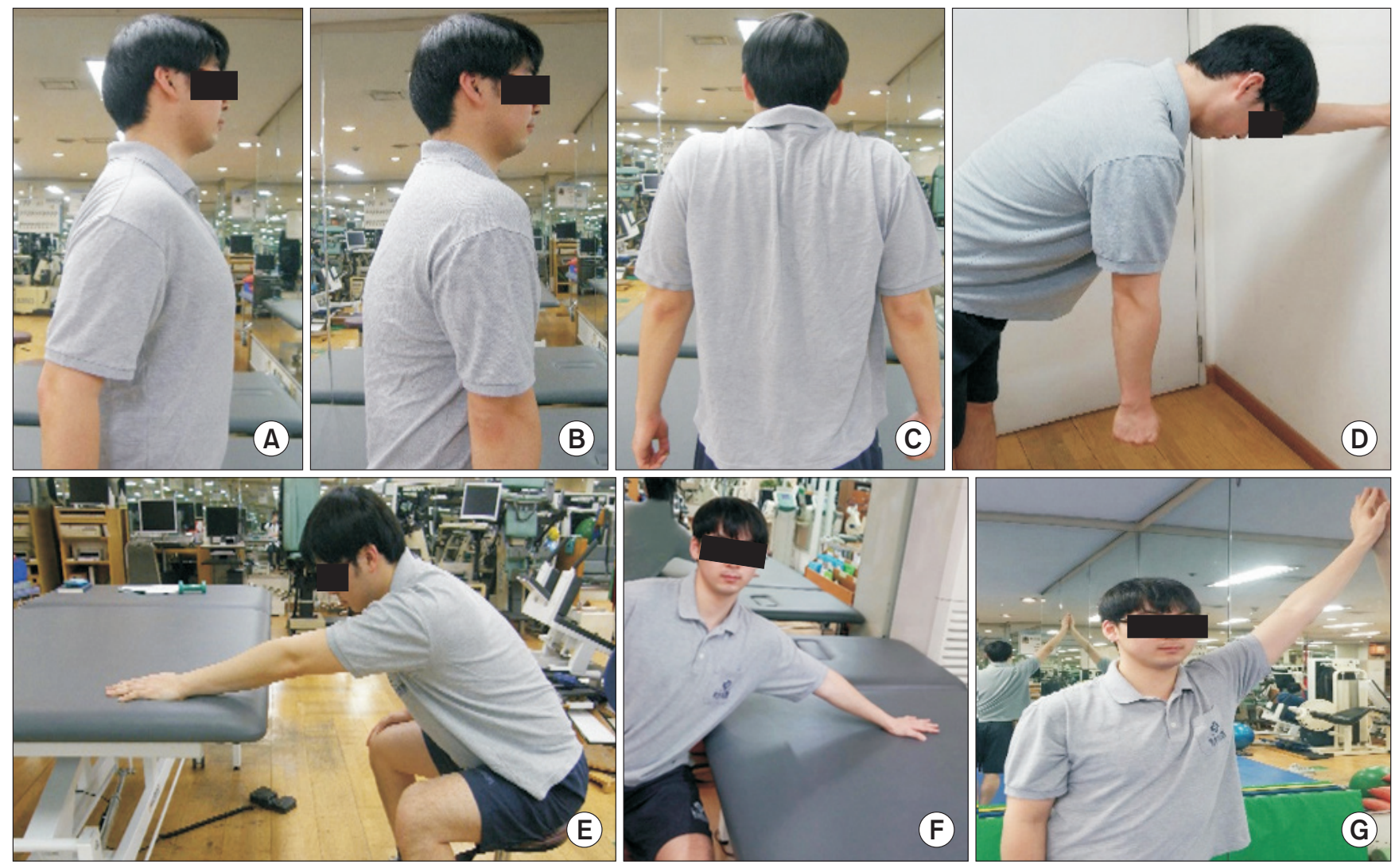

Fig. 1. Scapular mobilization exercise (A-C) and pendulum exercise (D) were conducted to improve the movement of the scapula before the range of motion exercise. Next, the table slide exercise for flexion (E), abduction (F), and wall climb exercise $(\mathrm{G})$ were conducted. All the pictures were contained in the leaflet distributed. 
supervised rehabilitation were included as the home exercise group. More than one education session as well as a leaflet was provided for the home exercise group.

The tear size was measured intraoperatively and categorized using the DeOrio and Cofield classification. ${ }^{13)}$ Single row repair and suture bridge technique were used on a case by case basis, based on the tear pattern, the quality of the tissue, and tension of the tendon. Additional side-to-side repair and biceps procedure (tenotomy or tenodesis) was performed, if necessary.

The IRB approved a request to waive the documentation of informed consent.

\section{Rehabilitation Exercise}

The involved shoulder was immobilized for four weeks after arthroscopic rotator cuff repair in all patients, using a sling with a small pillow. Elbow and wrist were allowed motion right after the surgery. Rehabilitation exercise started at Week-5. A supervised rehabilitation exercise program was advised for all patients. Pa- tients who wanted home rehabilitation exercise were imparted proper education of the exercise program. The supervision and education of the rehabilitation exercise was carried out by two trainers, having an experience of more than 3 years. The group that conducted home rehabilitation exercise was compared as the control group to the supervised rehabilitation exercise group.

In the supervised rehabilitation exercise group, scapular mobilization and pendulum exercises were conducted to improve the movement of the scapula, from Week-5 through Week-8 after surgery (Fig. 1). For shoulder motion, passive motion exercise, including closed-chain overhead stretching, was started along with the other programs (Fig. 1). ${ }^{14)}$ Active assistive ROM exercise was gradually introduced under expert supervision, once the passive motion arc increased (Fig. 2). The rehabilitation program consisted of 30 minutes sessions, 3 times a week for 8 weeks. From Week-9, additional rehabilitation was conducted, but was limited to patients desirous of the same. The remaining patients continued their rehabilitation exercises at home.
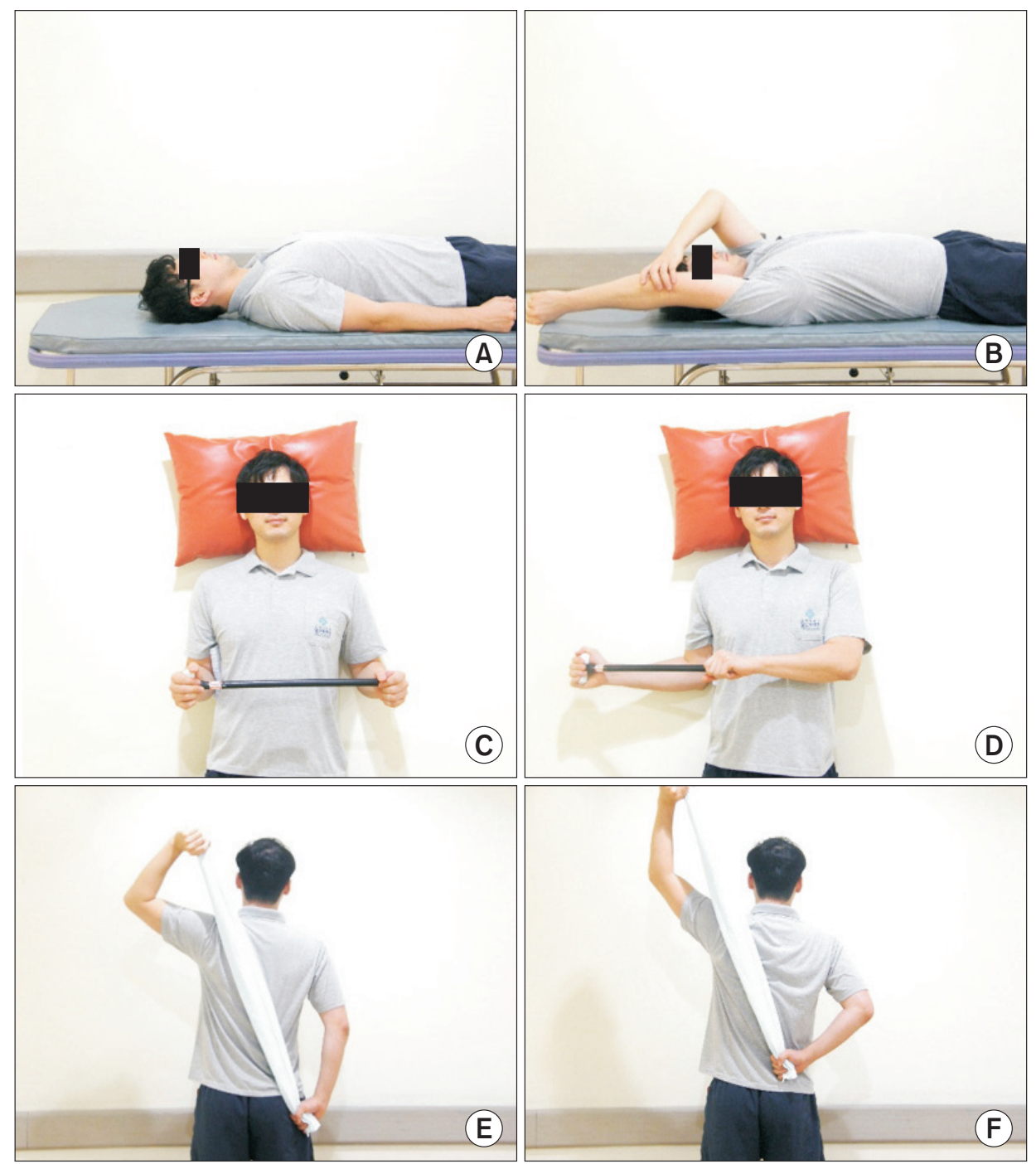

Fig. 2. Active assistive range of motion exercise for flexion (A, B), external rotation $(\mathrm{C}, \mathrm{D})$, and internal rotation $(\mathrm{E}, \mathrm{F})$ was gradually allowed as the passive motion arc increased. All the pictures were contained in the leaflet distributed. 
At Week-5 after the surgery, the home rehabilitation exercise group was similarly imparted details of 30 minutes of rehabilitation exercise training, and a leaflet guide was provided regarding the postoperative rehabilitation exercise. Those who had opted for home exercise had the freedom to return to the supervised rehabilitation program, and also obtain help for the rehabilitation exercise at home, if required. Home rehabilitation exercise also continued until 3 months after the surgery.

\section{Evaluation}

Pain level was assessed using the Numerical Rating Scale (NRS). No pain was scored as 0 , and unbearabe/most severe pain was 10. For functional evaluation, the Single Assessment Numeric Evaluation (SANE) ${ }^{15)}$ questionnaire assessed the patients for their current shoulder function, where 100 points indicated normal. Pain NRS, SANE score, and ROM were measured prior to surgery, at Week-5 after surgery (before the start of rehabilitation), at Week-9 after surgery (after rehabilitation exercise for four weeks), and at 6 months after surgery.

The shoulder ROM was measured by a single exercise therapist, who was independent from the surgery. Using a goniometer, the passive ROM was measured for forward flexion, external rotation and abduction, internal rotation, and digitization of the level of thumb reach by internal rotation of the arm to the back (1-12: T1-T12 vertebrae, 13-17: L1-L5 vertebrae, 18: sacrum, and 19: greater tuberosity of the femur).

\section{Statistical Analysis}

For statistical analysis of this study, we used the IBM SPSS Statistics for Windows, ver. 21.0 (IBM Co., Armonk, NY, USA). All data were indicated as mean \pm standard deviation. To compare the differences in the measured values between supervised rehabilitation and home rehabilitation exercise groups, an independent t-test was used for data with normal distribution. MannWhitney test was used for the non-parametric data. Paired t-test was used for comparing the measured values before and after the surgery in each group. To evaluate the correlation between groups for ROM recovery, pain, and function score, a Pearson correlation test was conducted and verified, and the significance level for statistical verification was set at 0.05 .

\section{Results}

Totally, 49 participants completed the study, between June 2015 and February 2017. The supervised rehabilitation exercise group included 29 patients, whereas the home rehabilitation exercise group enrolled 20 patients. None of the patients switched from the home rehabilitation to supervised group. The average age of the supervised rehabilitation exercise group was $59.8 \pm$ 6.6 years, while that of the home rehabilitation exercise group was $59.5 \pm 10.4$ years. There were 14 men and 15 women in the supervised rehabilitation exercise group, and 10 men and 10 women in the home rehabilitation exercise group.

There were no differences in the demographic data, including symptom duration, dominant arm, involved arm, mean height, and weight, between the two groups (Table 1).

Of the 49 patients, the rotator cuff tears were classified as 4 partial, 8 small, 28 medium, and 9 large tears. There was no difference between the two groups in terms of tear size $(p=0.200)$ (Table 1).

For the subjective pain NRS, no difference was observed between the supervised and home rehabilitation exercise groups at Week-5 after the surgery $(4.1 \pm 2.3$ and $4.5 \pm 2.5$, respectively; $p=0.574)$ and at Week-9 after the surgery $(3.5 \pm 2.0$ and $3.0 \pm$ 1.8 , respectively; $p=0.334$ ) (Table 2 ).

The SANE score also showed no difference between the two groups at Week-5 $(43.8 \pm 17.4$ and $42.0 \pm 16.4$, respectively; $p=0.716$ ). However, at Week-9 after the surgery, the supervised rehabilitation exercise group revealed a statistically significant higher score than the home rehabilitation exercise group (63.8 \pm 15.0 and $55.0 \pm 13.6$, respectively; $p=0.038$ ) (Table 2).

No significant differences were observed in the ROM evalu-

Table 1. Baseline Characteristics of Participants

\begin{tabular}{lccc}
\hline \multicolumn{1}{c}{ Characteristic } & Supervised exercise group $(\mathrm{n}=29)$ & Home exercise group $(\mathrm{n}=20)$ & $p_{\text {-value }}^{*}$ \\
\hline Age (yr) & $59.8 \pm 6.6$ & $59.5 \pm 10.4$ & 0.806 \\
\hline Sex (male/female) & $14 / 15$ & $10 / 10$ & 0.908 \\
\hline Dominance (right/left) & $19 / 10$ & $17 / 3$ & 0.116 \\
\hline Affected (right/left) & $19 / 10$ & $11 / 9$ & 0.473 \\
\hline Duration of symptom (mo) & $11.6 \pm 14.9$ & $14.0 \pm 27.2$ & 0.717 \\
Height (cm) & $162.2 \pm 5.9$ & $162.8 \pm 7.9$ & 0.858 \\
\hline Weight (kg) & $63.6 \pm 9.4$ & $63.1 \pm 10.2$ & 0.784 \\
\hline Tear size (partial/small/medium/large) & $2 / 6 / 17 / 4$ & $2 / 2 / 11 / 5$ & 0.200 \\
\hline
\end{tabular}

Values are presented as mean \pm standard deviation or number only.

${ }^{*}$ There were no significant differences between the two groups. 
Table 2. Value for Passive Range of Motion in Degrees, Pain NRS and SANE for Each Group

\begin{tabular}{|c|c|c|c|c|c|c|c|}
\hline \multirow{2}{*}{ Variable } & \multirow{2}{*}{ Baseline } & \multirow{2}{*}{ Week-5 } & \multirow{2}{*}{ Week-9 } & \multirow{2}{*}{ Week-9 minus week-5 } & \multicolumn{3}{|c|}{ Supervised vs. home exercise group } \\
\hline & & & & & Week-5 & Week-9 & Week- 9 minus week- 5 \\
\hline $\mathrm{FF}\left(^{\circ}\right)$ & & & & & $3.2(p=0.700)$ & $8.5(p=0.274)$ & $3.2(p=0.636)$ \\
\hline Supervised & $144.3 \pm 31.2$ & $109.5 \pm 28.8$ & $146.2 \pm 22.9$ & $36.7 \pm 18.4$ & & & \\
\hline Home & $150 \pm 19.7$ & $106.3 \pm 28.6$ & $137.8 \pm 28.2$ & $33.5 \pm 26.0$ & & & \\
\hline $\operatorname{ER}\left({ }^{\circ}\right)$ & & & & & $-12.3(p=0.200)$ & $4.0(p=0.455)$ & $8.3(p=0.018)$ \\
\hline Supervised & $51.4 \pm 14.1$ & $15.7 \pm 15.7$ & $33.3 \pm 18.6$ & $17.6 \pm 12.7$ & & & \\
\hline Home & $50.3 \pm 26.1$ & $28.0 \pm 18.5$ & $37.3 \pm 17.8$ & $9.3 \pm 10.9$ & & & \\
\hline $\operatorname{IR}\left({ }^{\circ}\right)$ & & & & & $0.9(p=0.369)$ & $0.6(p=0.585)$ & $-0.3(p=0.762)$ \\
\hline Supervised & $11.6 \pm 4.9$ & $17.3 \pm 2.7$ & $13.8 \pm 3.9$ & $-3.5 \pm 2.6$ & & & \\
\hline Home & $10.8 \pm 5.0$ & $16.4 \pm 3.6$ & $13.2 \pm 4.1$ & $-3.8 \pm 3.3$ & & & \\
\hline Abduction $\left(^{\circ}\right)$ & & & & & $1.2(p=0.897)$ & $4.1(p=0.699)$ & $8.1(p=0.521)$ \\
\hline Supervised & $131.4 \pm 41.2$ & $93.4 \pm 32.4$ & $122.9 \pm 37.9$ & $29.5 \pm 19.9$ & & & \\
\hline Home & $133.0 \pm 33.3$ & $92.3 \pm 31.0$ & $127.0 \pm 34.5$ & $34.8 \pm 32.2$ & & & \\
\hline NRS & & & & & $-0.4(p=0.574)$ & $0.5(p=0.334)$ & $0.1(p=0.889)$ \\
\hline Supervised & $6.3 \pm 2.6$ & $4.1 \pm 2.3$ & $3.5 \pm 2.0$ & $-1.7 \pm 1.5$ & & & \\
\hline Home & $6.3 \pm 1.7$ & $4.5 \pm 2.5$ & $3.0 \pm 1.8$ & $-1.7 \pm 2.0$ & & & \\
\hline SANE & & & & & $1.8(p=0.716)$ & $8.8(p=0.038)$ & $6.0(p=0.094)$ \\
\hline Supervised & $54.1 \pm 23.1$ & $43.8 \pm 17.4$ & $63.8 \pm 15.0$ & $20.0 \pm 11.6$ & & & \\
\hline Home & $57.5 \pm 19.2$ & $42.0 \pm 16.4$ & $55.0 \pm 13.6$ & $14.0 \pm 12.3$ & & & \\
\hline
\end{tabular}

Values are presented as mean \pm standard deviation.

NRS: Numerical Rating Scale, SANE: Single Assessment Numeric Evaluation, FF: forward flexion, Supervised: supervised exercise group, Home: exercise group, ER: external rotation, IR: internal rotation.

Table 3. Value for Passive Range of Motion in Degree, Pain NRS and SANE for Each Group at 6 Month after Surgery

\begin{tabular}{|c|c|c|c|c|c|c|}
\hline Variable & $\mathrm{FF}\left(^{\circ}\right)$ & $\operatorname{ER}\left(^{\circ}\right)$ & $\operatorname{IR}\left({ }^{\circ}\right)$ & Abduction $\left({ }^{\circ}\right)$ & NRS & SANE \\
\hline Supervised & $156.6 \pm 19.9$ & $37.2 \pm 22.2$ & $8.9 \pm 2.3$ & $140.0 \pm 34.6$ & $1.9 \pm 1.58$ & $70.0 \pm 16.3$ \\
\hline Home & $157.5 \pm 18.9$ & $32.3 \pm 13.2$ & $10.2 \pm 3.1$ & $131.5 \pm 35.3$ & $2.5 \pm 1.6$ & $68.5 \pm 15.9$ \\
\hline$p$-value & 0.868 & 0.373 & 0.110 & 0.406 & 0.267 & 0.751 \\
\hline
\end{tabular}

Values are presented as mean \pm standard deviation.

NRS: Numerical Rating Scale, SANE: Single Assessment Numeric Evaluation, FF: forward flexion, ER: external rotation, IR: internal rotation, Supervised: supervised exercise group, Home: exercise group.

ated at Week-5 (prior to rehabilitation) or Week-9 (post rehabilitation): forward flexion $(p=0.700,0.274$, respectively), external rotation $(p=0.200,0.455$, respectively), internal rotation $(p=0.369,0.585$, respectively) and abduction $(p=0.897,0.699$, respectively) (Table 2). However, a comparison in the improvement in each ROM measured revealed a significant difference in external rotation between the supervised rehabilitation exercise group $\left(17.6^{\circ} \pm 12.7^{\circ}\right)$ and the home rehabilitation exercise group $\left(9.3^{\circ} \pm 10.9^{\circ}\right)$ from Week-5 to Week-9 $(p=0.018)$ (Table 2). Other variables showed no differences. Overall, there were no significant differences between the two groups in ROM, pain NRS and SANE at 6 months after surgery (Table 3).
Table 4. Correlation between Increased Range of Motion and Reduced NRS

\begin{tabular}{lcc}
\hline Variable & $\begin{array}{c}\text { Pearson's correlation } \\
\text { coefficient }\end{array}$ & $p$-value \\
\hline Forward flexion & 0.032 & 0.828 \\
\hline External rotation & -0.255 & 0.077 \\
\hline Internal rotation & 0.068 & 0.642 \\
\hline Abduction & -0.188 & 0.196 \\
\hline
\end{tabular}

NRS: Numerical Rating Scale. 
No statistically significant correlation was observed between the increases of forward flexion, external rotation, internal rotation, and abduction with pain relief (Table 4). However, increased external rotation correlated with increased SANE between Week-5 and Week-9 (Pearson correlation coefficient $=0.286, p=0.047$ ).

\section{Discussion}

In the current study, the recovery of external rotation was faster in the supervised exercise group than in the home exercise group, although forward flexion, internal rotation, and abduction showed no difference. In addition, the SANE score was higher in the supervised exercise group at Week-9, i.e., one month after the rehabilitation. Pain NRS showed no correlation with ROM improvement, and other variables revealed no differences between the two groups.

Immobilization for a certain period and proper rehabilitation are important factors for successful clinical results in rotator cuff repair surgery. However, a longer period of immobilization might lead to higher prevalence of stiffness, despite a lower possibility of retear. ${ }^{16)}$ It was reported that the prevalence of shoulder stiffness after an arthroscopic rotator cuff repair was usually $10 \%$, and the resistant postoperative stiffness (which is permanent or needs capsular release) was 3.3\% to $4.5 \%{ }^{17)}$ Even patients with no complaints of long term stiffness show a slow improvement in pain, ROM, and function up to 12 months after the surgery. ${ }^{18)}$

Since most shoulder surgeons prefer to immobilize the operated shoulder for a short duration to protect repair, rehabilitation exercise is greatly emphasized to prevent persistent stiffness and facilitate early recovery. Many studies have reported that passive ROM exercise is effective for preventing shoulder stiffness after rotator cuff repair. ${ }^{4,19-21)}$ Rehabilitation exercises are generally prescribed and conducted systematically under the supervision of an exercise specialist or physical therapist. However, some patients opt to do the rehabilitation exercises themselves due to factors such as time or cost limitation and inconvenience. Literature has cited mixed results for its effectiveness in the recovery of ROM for both supervised and home-based exercise. One study indicated the clinical result was better in the supervised rehabilitation exercise group, ${ }^{5)}$ whereas other studies showed no difference between the two groups. ${ }^{10,22)}$ Also, there are diverse opinions regarding the supervised and the home exercise protocols. While supervised exercise subjects the patient to both economic and temporal burdens, home-based exercise can reduce the overall health-related costs and time. ${ }^{23)}$ However, it is more likely that the patients who do home exercise might not be devoted to their regime, and ROM gain might not be as effective as when supervised. In our study, the external rotation decreased between Week-8 and 6 months after surgery in the home rehabilitation exercise group. As the pain decreased, pa- tients tend not to exercise enough. Also, the increased follow-up interval could have affected this result.

We found no statistically significant difference between the two groups in terms of ROM, except for external rotation. Patients were generally cooperative in rehabilitation during the first few weeks. However, one interesting finding was that the increase in external rotation from Week-5 to Week-9 was significantly higher in the supervised exercise group. Also, the SANE score was higher in the supervised group as compared to home exercise group.

In the home exercise group, patients could perform most of the exercises rather easily, including table slide for forward flexion, or hand behind the waist for internal rotation. However, the external rotation exercise using a T-bar was often mixed with abduction motion. Thus, the exercise performed for external rotation was frequently not as how the surgeon intended. Under supervised rehabilitation, the patients were trained to perform the exercise correctly by constant retraining. In addition, the supervised routinely included exercises for stabilization and mobilization of the scapula, prior to passive motion exercise. This might reflect in the higher SANE score in the supervised exercise group, which could be an outcome from the increased external rotation range of this group.

Our study was unable to reveal a correlation between the ROM recovery and pain reduction. We postulated that the more rapid and effective recovery of ROM leads to pain reduction or effective pain control, and also that patients with less pain showed more rapid or improved ROM that those with more pain. However, due to the small sample size and other compounding factors such as use of analgesics, our study failed to prove the relationship between the ROM and pain.

This study has a few limitations. First, this study has the inherent weakness of being a retrospective study with a small sample size. Second, although the purpose of this study was to compare the short-term recovery of both rehabilitation exercise groups, an accompanying evaluation of the long-term outcome would have enhanced our results. Third, the exercise frequency was not consistent in every patient, even though the prescribed exercise was 3 times a week for 4 weeks. Fourth, the home exercise group consisted of patients who had initially prescribed for supervised exercise, but later opted for home exercise. Fifth, patients were assessed only clinically, without any supporting radiologic or arthroscopic evaluation. Although this study is not a randomized trial.

\section{Conclusion}

After 4 weeks of rehabilitation post the arthroscopic rotator cuff repair, the supervised exercise group showed better improvement in external rotation and a higher SANE score. However, there was no statistically significant correlation between the 
recovery of ROM and pain relief in this short-term study.

\section{References}

1. Saccomanno MF, Sircana G, Cazzato G, Donati F, Randelli P, Milano G. Prognostic factors influencing the outcome of rotator cuff repair: a systematic review. Knee Surg Sports Traumatol Arthrosc. 2016;24(12):3809-19.

2. Abtahi AM, Granger EK, Tashjian RZ. Factors affecting healing after arthroscopic rotator cuff repair. World J Orthop. 2015; 6(2):211-20.

3. Hayes K, Ginn KA, Walton JR, Szomor ZL, Murrell GA. A randomised clinical trial evaluating the efficacy of physiotherapy after rotator cuff repair. Aust J Physiother. 2004;50(2):77-83.

4. Lee BG, Cho NS, Rhee YG. Effect of two rehabilitation protocols on range of motion and healing rates after arthroscopic rotator cuff repair: aggressive versus limited early passive exercises. Arthroscopy. 2012;28(1):34-42.

5. Lisinski P, Huber J, Wilkosz P, et al. Supervised versus uncontrolled rehabilitation of patients after rotator cuff repair-clinical and neurophysiological comparative study. Int J Artif Organs. 2012;35(1):45-54.

6. Senbursa G, Baltaci G, Atay A. Comparison of conservative treatment with and without manual physical therapy for patients with shoulder impingement syndrome: a prospective, randomized clinical trial. Knee Surg Sports Traumatol Arthrosc. 2007;15(7):915-21.

7. Bang MD, Deyle GD. Comparison of supervised exercise with and without manual physical therapy for patients with shoulder impingement syndrome. J Orthop Sports Phys Ther. 2000;30(3):126-37.

8. Şenbursa G, Baltaci G, Atay ÖA. The effectiveness of manual therapy in supraspinatus tendinopathy. Acta Orthop Traumatol Turc. 2011;45(3):162-7.

9. Chou CT, Hu W, Wen CS, Wang SF, Lieu FK, Teng JT. Efficacy of informed versus uninformed physiotherapy on postoperative retear rates of medium-sized and large rotator cuff tears. J Shoulder Elbow Surg. 2015;24(9):1413-20.

10. Büker N, Kitiş A, Akkaya S, Akkaya N. Comparison of the results of supervised physiotherapy program and home-based exercise program in patients treated with arthroscopic-assisted mini-open rotator cuff repair. Eklem Hastalik Cerrahisi. 2011; 22(3):134-9.

11. Krischak G, Gebhard F, Reichel H, et al. A prospective randomized controlled trial comparing occupational therapy with home-based exercises in conservative treatment of rotator cuff tears. J Shoulder Elbow Surg. 2013;22(9):1173-9.
12. Lafosse L, Jost B, Reiland Y, Audebert S, Toussaint B, Gobezie R. Structural integrity and clinical outcomes after arthroscopic repair of isolated subscapularis tears. J Bone Joint Surg Am. 2007;89(6):1184-93.

13. DeOrio JK, Cofield RH. Results of a second attempt at surgical repair of a failed initial rotator-cuff repair. J Bone Joint Surg Am. 1984;66(4):563-7.

14. Koo SS, Parsley BK, Burkhart SS, Schoolfield JD. Reduction of postoperative stiffness after arthroscopic rotator cuff repair: results of a customized physical therapy regimen based on risk factors for stiffness. Arthroscopy. 2011;27(2):155-60.

15. Williams GN, Gangel TJ, Arciero RA, Uhorchak JM, Taylor DC. Comparison of the Single Assessment Numeric Evaluation method and two shoulder rating scales. Outcomes measures after shoulder surgery. Am J Sports Med. 1999;27(2):214-21.

16. Back YW, Tae SK, Kim MK, Kwon OJ. The effect of different starting periods of passive exercise on the clinical outcome of arthroscopic rotator cuff repair. Clin Should Elbow. 2014;17(2):57-63.

17. Denard PJ, Lädermann A, Burkhart SS. Prevention and management of stiffness after arthroscopic rotator cuff repair: systematic review and implications for rotator cuff healing. Arthroscopy. 2011;27(6):842-8.

18. Cho $\mathrm{CH}$, Lim YJ. When does the quality of life improve after rotator cuff repair? J Korean Orthop Assoc. 2013;48(4):281-9.

19. Arndt J, Clavert P, Mielcarek P, Bouchaib J, Meyer N, Kempf JF; French Society for Shoulder \& Elbow (SOFEC). Immediate passive motion versus immobilization after endoscopic supraspinatus tendon repair: a prospective randomized study. Orthop Traumatol Surg Res. 2012;98(6 Suppl):S131-8.

20. Raab MG, Rzeszutko D, O'Connor W, Greatting MD. Early results of continuous passive motion after rotator cuff repair: a prospective, randomized, blinded, controlled study. Am J Orthop (Belle Mead NJ). 1996;25(3):214-20.

21. Koo SS, Burkhart SS. Rehabilitation following arthroscopic rotator cuff repair. Clin Sports Med. 2010;29(2):203-11, vii.

22. Roddey TS, Olson SL, Gartsman GM, Hanten WP, Cook KF. A randomized controlled trial comparing 2 instructional approaches to home exercise instruction following arthroscopic full-thickness rotator cuff repair surgery. J Orthop Sports Phys Ther. 2002;32(11):548-59.

23. Aytar A, Baltaci G, Uhl TL, Tuzun H, Oztop P, Karatas M. The effects of scapular mobilization in patients with subacromial impingement syndrome: a randomized, double-blind, placebo-controlled clinical trial. J Sport Rehabil. 2015;24(2):11629. 